\title{
Comparação do ensaio de inteferon-gamma e teste cutâneo tuberculínico para diagnóstico de tuberculose latente em profissionais de saúde de um centro de referência terciária para tuberculose do Estado da Bahia
}

\author{
Comparation of the interferon gamma release assay and tuberculin skin test for \\ diagnosis of latent tuberculosis in health professionals at a tertiary tuberculosis \\ reference center, State of Bahia
}

Marilda Casela ${ }^{1 *}$

${ }^{1}$ Farmacêutica bioquímica. Doutora em Processos Interativos dos Órgãos e Sistemas.

\begin{abstract}
Resumo
Introdução: o Teste Cutâneo Tuberculínico (TCT) tem sido utilizado como diagnóstico para a Tuberculose Infecção Latente (TBIL). Os profissionais de saúde, principalmente os que trabalham com assistência a pacientes com tuberculose (TB), têm risco elevado de contrair a doença ou a TBIL. Normas de biossegurança e qualificação da equipe de trabalho são fundamentais para o controle da Tb entre os profissionais de saúde. Atualmente testes diagnósticos mais específicos para a TBIL têm sido utilizados como alternativa para o padronizado TCT, dentre eles o QuantiFERON-TB Gold $\left(\mathrm{QTF}^{\circ}\right)$. Objetivo: comparar e avaliar os resultados obtidos no QTF ${ }^{\circ}$ e Teste Cutâneo Tuberculínico dos profissionais de saúde de um centro de referência terciário para Tuberculose. Metodologia: o projeto foi aprovado pelo Comitê de Ética em Pesquisa da Fundação Bahiana Desenvolvimento Científico e pelo Comitê de Ética em Pesquisa da instituição hospitalar. Foi utilizado o banco de dados do setor de medicina ocupacional da Instituição e os 87 voluntários que atenderam aos critérios do estudo foram categorizados e distribuídos em dois grupos: TCT negativo $(<5 \mathrm{~mm})$ e TCT positivo ( $>5 \mathrm{~mm})$. Foi realizada coleta de sangue para o teste QTF $^{\circ}$. Resultados: dos 47 profissionais de saúde TCT negativo $53,2 \%$ tiveram resultado QTF negativo. Dos 40 profissionais TCT positivo, 67,5\% apresentaram resultado QTF $^{\circ}$ positivo. $O$ grau de concordância do índice kappa foi de 0,24. Conclusão: O QTF ${ }^{\circ}$ não mostrou uma especificidade superior quando comparado ao TCT.

Palavras-chave: Prevenção \& controle. Tuberculose Latente. Pessoal de Saúde. Interferon Gama. Teste Tuberculínico.
\end{abstract}

\begin{abstract}
Introduction: tuberculin Skin test (TST) is a test used to Latent tuberculosis Infection (LTBI) diagnosis. Health professional, principally those that work with tuberculosis (TB) patients, have a high risk of contract and developing the disease or LTBI. Biosecurity norms and professional qualification are fundamentals to TB control in care centers. Actually some diagnosis tests with higher specificity of LTBI are used as an alternative to the TST, such as QuantiFERON-TB Gold $\left(Q_{T} F^{\circ}\right)$. Objective: to compare and evaluate the results obtained in the Quantiferon-TB Gold ${ }^{\circledR}$ and TST, of health professionals from a tertiary referral center for Tuberculosis. Methodology: the project was approved by a Research Ethics Committee of FBDC, and by the CEP of the hospital institution. The data base of the occupational medicine sector and the health professionals were categorized and distributed in two groups: Negative Tuberculin test and Positive Tuberculin test. The 93 volunteers who met the criteria and agreed to participate, signed the consent form, with subsequent data collection. A blood collection was also performed to perform Quantiferon-TB Gold ${ }^{\circledR}$ test. Results: of the 47 PS negative Tuberculin test, $53.2 \%$ had negative Quantiferon-TB Gold ${ }^{\circledR}$ results and $46.8 \%$ positive. Of the health professional positive Tuberculin test, $32.5 \%$ presented negative Quantiferon-TB Gold ${ }^{\circledR}$ result and $67.5 \%$ positive. Conclusion: quantiferon-TB Gold ${ }^{\circledR}$ did not show superior specificity when compared to Tuberculin test on this coorte.
\end{abstract}

Keywords: Prevention \& control. Latent Tuberculosis. Health Personnel. Interferon Gamma. Tuberculin Test.

\section{INTRODUÇÃO}

Profissionais de saúde (PS) de centros de referência para tuberculose (TB) encontram-se sob risco elevado de infecção e/ou adoecimento por esta doença devido à exposição direta a pacientes que podem estar em fase

Correspondente/Corresponding: *Marilda Casela - Instituto de Ciências da Saúde/LABIMUNO-UFBA/Salvador-Ba - End: Av. Reitor Miguel Calmon s/n Vale do Canela Salvador - Ba - Tel : (71) 99232-4352 - E-mail: marildacasela@hotmail.com bacilífera ${ }^{1}$. Por conta disso, mesmo que não apresentem sintomas, podem ter a infecção de forma latente. Entretanto, os dados sobre a tuberculose infecção latente (TBIL) no Brasil ainda são escassos em razão da inexistência da obrigatoriedade de notificação.

A TBIL compreende o período entre a entrada do bacilo no organismo e o aparecimento da doença, sendo que não são evidenciados sinais e sintomas clínicos e radiológicos ${ }^{2}$. 
Até 2010, apenas alguns casos de TBIL eram tratados com quimioprofilaxia, mas a partir deste ano a indicação foi ampliada, com o objetivo de evitar adoecimento para aquelas pessoas com maior risco de desenvolver a TB, como os profissionais de saúde ${ }^{1}$.

Estima-se que um terço da população mundial esteja infectada por Mycobacterium tuberculosis (MTB), incluindo TB doença e TBIL. Desde 2015, a Organização Mundial da Saúde intensificou a coleta de dados relacionados com a TBIL em países com uma taxa de incidência menor do que 100/100.000 habitantes. No trabalho foi enfatizada a importância da realização de um estudo especial das políticas públicas e práticas existentes ${ }^{3}$.

O Teste Cutâneo Tuberculínico (TCT) é o exame padronizado no Brasil para o diagnóstico da TBIL. Esse teste avalia a resposta imune celular in vivo contra extrato antigênico do MTB. Nesse teste é utilizada proteína pruficada derivada de Mycobacterium tuberculosis na concentração de 2 unidades de tuberculina (PPD-RT 232 UT) de procedência dinamarquesa (Statens Serum institut de Copenhague/Dinamarca). A solução de PPD - RT 23 deve ser aplicada por meio de injeção intradérmica, na dose de 0,1 $\mathrm{ml}$ (0,04 mcg), no terço médio da face anterior do antebraço esquerdo, evitando-se a inoculação em áreas com lesões, veias superficiais, cicatrizes ou tatuagens. , a prova tuberculínica provoca a formação de uma pápula de 7 a $8 \mathrm{~mm}$ de diâmetro, com limites precisos, pálida e de aspecto pontilhado, que persiste por alguns minutos após a aplicação.

Para a realização da leitura do TCT verificar se houve aparecimento de uma região endurecida no local da aplicação e medir com uma régua a área de enduração no sentido transversal em relação ao eixo longitudinal do antebraço. A leitura do TCT é registrada em milímetros após 48 a 72 horas, a reação provocada pela proteína tuberculínica se caracteriza por uma hipersensibilidade cutânea tardia, tipo IV4.

No entanto, o teste apresenta limitações como a necessidade de retorno do paciente ao estabelecimento de saúde para realizar a leitura, baixa sensibilidade especialmente em indivíduos imunocomprometidos, baixa especificidade, decorrente da possibilidade do resultado falso positivo em populações que tenham ampla cobertura vacinal com BCG, assim como reação cruzada com micobactérias atípicas4.

As proteínas antigênicas micobacterianas como Early Secretory antigen Target-6 (ESAT-6), antigens tuberculosis (TB7.7) e culture filtrate protein-10 (CFP-10), específicas e expressas pelo complexo M. tuberculosis, possibilitaram o desenvolvimento de testes diagnósticos mais específicos para infecção por MTB. Dentre estes o QuantiFERON-TB-Gold ${ }^{\oplus}\left(\mathrm{QTF}^{\circ}\right.$ ): exame laboratorial de sangue, in vitro baseado na dosagem de IFN- $\gamma$ que não requer retorno do paciente ao laboratório para leitura, resultado disponível em 24 horas que deve ser interpretado também em conjunto com a avaliação de risco, radiografia do tórax, avaliações clínicas e de diagnóstico para a TBIL e TB doença ${ }^{5}$.
Além disso, o teste apresenta especificidade acima de $98 \%$, contra $35,4 \%$ do TCT, usando um ponto de corte de $10 \mathrm{~mm}$ em indivíduos saudáveis vacinados com BCG $^{5}$.

O risco de transmissão nosocomial (transmitida em ambiente de trabalho) de MTB varia em função principalmente da prevalência de doentes no ambiente de trabalho e da efetividade do programa de controle da infecção da instituição. Assim, o diagnóstico e tratamento precoces, como medidas preventivas, administrativas, ambientais e individuais podem reduzir o risco de contágio ocupacional da $\mathrm{TB}^{6}$.

O objetivo deste estudo foi comparar e avaliar os resultados obtidos no TCT e QTF ${ }^{\circledR}$ de profissionais de saúde de um centro de referência terciário para TB.

\section{METODOLOGIA}

\section{Aspectos Éticos}

O presente projeto foi aprovado pelo Comitê de Ética em Pesquisa (CEP) da Fundação Bahiana para o Desenvolvimento das Ciências (FBDC) sob $n$ de CAAE: 57274316.3.0000.5544de acordo com as normas vigentes na Resolução CNS n‥ 196/96 e com adendo/emenda aprovado em 11/2011. Também foi submetido e aprovado pelo Comitê de Ética em Pesquisa da instituição hospitalar da qual foi realizado o estudo.

\section{Desenho do Estudo}

Trata-se de um estudo transversal, observacional, retrospectivo, de base hospitalar com abordagem descritiva, desenvolvido em uma unidade hospitalar de referência terciária para tuberculose em uma capital do nordeste brasileiro.

\section{População de Estudo}

Os 93 profissionais de saúde de serviço TB que atenderam aos critérios de inclusão e que aceitaram participar do estudo assinaram o termo de consentimento livre e esclarecido. Em seguida, foi realizada a coleta de dados sociodemográfico e laboratorial.

\section{Coleta de dados}

Foi utilizado o banco de dados do setor de medicina ocupacional do referido hospital. Os profissionais de saúde foram categorizados e distribuídos em dois grupos: Grupo TCT negativo com resultado $<5 \mathrm{~mm}$ e Grupo TCT positivo com resultado $>10 \mathrm{~mm}$ (neste último grupo os indivíduos foram selecionados de maneira aleatória).

\section{Teste Cutâneo Tuberculínico}

Foram utilizados os seguintes critérios para o TCT: Resultado referente ao último periódico (últimos 12 meses e/ou aplicação do TCT com leitura após 72 horas). Todos os profissionais do estudo tinham contato com pacientes TB à no mínimo 10 anos. De todos os participantes 
foi realizado o exame de hemograma para a verificação de alterações hematológicas e PCR ultra sensível como marcador de processo inflamatório. De acordo com estes parâmetros foram excluídos 6 participantes por apresentarem alterações no PCR.

\section{Ensaio de Liberação de IFN-gama (IGRA)-teste QTF}

Para realização do teste $\mathrm{QTF}^{\circ}$ foi coletado um volume de $3 \mathrm{~mL}$ de sangue, e a execução e análise do QFT foram realizadas conforme orientação do fabricante.

A partir da análise dos resultados do TCT e do IGRA, foi possível gerar informações sob a forma de gráficos e tabelas.

\section{Análise estatística}

A análise estatística foi realizado para obtenção do índice de concordância Kappa através do software GraphPad Prism.

\section{RESULTADOS}

A média de idade dos dois grupos de profissionais de saude estudados foi de 50 anos. A caracterização dos profissionais dos dois grupos está descrita na tabela 1 , com idade média, tempo médio de exposição, sexo e classificação étnica que foi auto referenciada segundo o IBGE. Do total de 87 PS, o predomínio foi do grupo de enfermagem (técnicos e enfermeiros) $(37,6 \%)$, seguidos de técnicos de laboratório (13,98\%), técnicos de radiologia $(8,6 \%)$, farmacêutico-bioquímicos $(7,5 \%)$ e outros profissionais $(32,26 \%)$, como mostrado na figura 1 .

Tabela 1 - Caracterização sociodemográfica dos PS no período de junho de 2014 a setembro de 2015. Salvador/BA, 2015.

\begin{tabular}{|ccc|}
\hline Características & $\begin{array}{c}\text { Grupo TCT } \\
\text { de repetição } \\
\text { negativo }<\mathbf{~ m m} \\
\text { Anos } \pm \mathrm{DP}\end{array}$ & $\begin{array}{c}\text { Grupo TCT positivo } \\
\mathbf{1 0} \mathbf{~ m m}\end{array}$ \\
\hline Idade & $52 \pm 5$ & Anos $\pm \mathrm{DP}$ \\
\hline Feminino & $45 \pm 10$ & $50 \pm-5$ \\
\hline Masculino & $\mathrm{n}(\%)$ & $52 \pm 8$ \\
\hline Sexo & $41(85)$ & $\mathrm{n}(\%)$ \\
\hline Feminino & $7(15)$ & $40(89)$ \\
\hline Masculino & $\mathrm{N}$ & $5(11)$ \\
\hline Classificação étnica & 20 & $\mathrm{n}$ \\
\hline Branco & 17 & 10 \\
\hline Pardo & 11 & 17 \\
\hline Negro & 0 & 17 \\
\hline Indígena & & 1 \\
\hline Contato & Anos $\pm \mathrm{DP}$ & Anos $\pm \mathrm{DP}$ \\
\hline Total (n=93) & $18 \pm 7,2$ & $22 \pm 5$ \\
\hline
\end{tabular}

TCT: Teste Cutâneo Tuberculínico/DP: Desvio Padrão

Fonte: Dados da pesquisa
Dos 47 profissionais de saúde TCT $<5 \mathrm{~mm}, 25(53,2 \%)$ tiveram resultado do QTF negativo e $22(46,8 \%)$ positivo. Dos 40 profissionais de saúde TCT positivo $>10 \mathrm{~mm}, 13$ $(32,5 \%)$ apresentaram resultado do QTF negativo e 27 $(67,5 \%)$ positivo, conforme tabela 2.

Tabela 2 - Comparação entre os resultados de teste cutâneo tuberculínico (TCT) e ensaio de detecção de interferon gama (IGRA). Salvador/BA, 2015.

\begin{tabular}{lccc}
\multicolumn{1}{c}{ Variáveis } & $\begin{array}{c}\text { IGRA negativo } \\
\mathbf{n} / \%\end{array}$ & $\begin{array}{c}\text { IGRA positivo } \\
\text { n/\% }\end{array}$ & TOTAL \\
\hline $\begin{array}{l}\text { TCT negativo de } \\
\text { repetição }\end{array}$ & $25 / 53,2$ & $22 / 46,8$ & 47 \\
TCT positivo & $13 / 32,5$ & $27 / 67,5$ & 40 \\
\hline
\end{tabular}

TCT: Teste Cutâneo Tuberculínico/IGRA: Ensaio de liberação de Interferon Gama

Outro dado digno de nota, foi o resultado do IGRA negativo em um participante com reação TCT flictenular $(25 \mathrm{~mm})$, a amostra de sangue foi coletada no ato da leitura do TCT, sem intervalo de tempo, para excluir qualquer dúvida. Posteriormente, esse PS foi encaminhado ao serviço ocupacional para avaliação clínica.

Gráfico 1 - Discriminação dos PS participantes do estudo por categoria profissional. Salvador/BA, 2015.

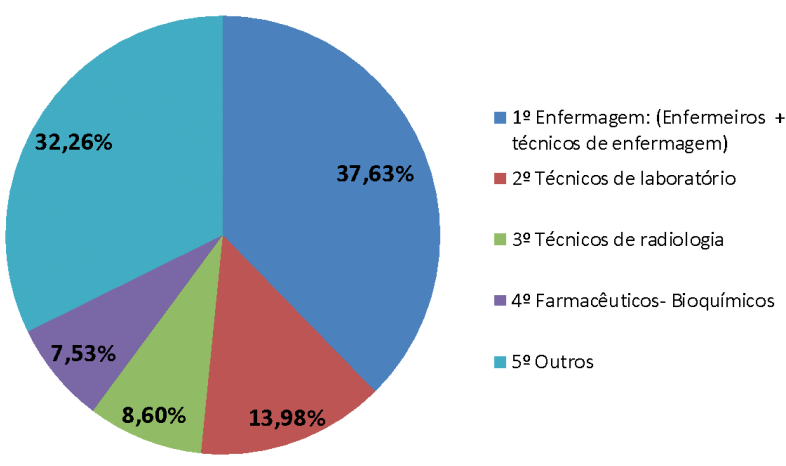

Fonte: Dados da pesquisa

Foi observado no presente estudo uma concordância "razoável" entre os testes, interpretada com o valor de índice Kappa de 0,24 e intervalo de confiança de 95\% $(0,003-0,404)$.

\section{DISCUSSÃO}

De acordo com a Organização Mundial de Saúde uma das formas mais eficazes para o controle da TB ativa é a identificação e o tratamento da TBIL. Na busca de um diagnóstico mais sensível e específico, novas tecnologias para substituir o TCT vêm sendo testado para detecção de TBIL. De acordo com a literatura nem todos os infectados com o bacilo desenvolvem a TB ativa, certos grupos têm 
um risco mais elevado de infecção por MTB, dentre esses, os profissionais de saúde ${ }^{7}$.

No Brasil, o teste tuberculínico é o padrão-ouro para diagnóstico da infecção de TBIL que mede as respostas imunes ao PPD in vivo. Uma das maiores limitações do TCT é a baixa especificidade, especialmente em indivíduos vacinados pelo BCG, ou naqueles infectados por micobactérias atípicas ou ambientais. $O$ teste pode evidenciar ainda sensibilidade baixa, especialmente em indivíduos imunocomprometidos. Outra limitação importante é a necessidade do paciente retornar ao laboratório no período de 48 a 96 horas, após a aplicação do PPD para leitura do resultado 8 .

Atualmente está disponível um novo teste de diagnóstico da infecção de TB in vitro, o IGRA, que é um exame de sangue controlado capaz de detectar a infecção TB com especificidade e sensibilidade maiores que a do TCT. Em 2014, a Agência Nacional de Vigilância Sanitária liberou o teste do IGRA para diagnóstico no Brasil. O uso anterior a essa data estava autorizado somente para pesquisa. No presente estudo foi utilizado o $\mathrm{QFT}^{\circledR}$ para a dosagem de IFN- $\gamma$ pelo método ELISA. Os dados do IGRA das amostras com valores diferentes aos encontrados nos registros de TCT foram reavaliados ou repetidos.

No nosso estudo a média de idade dos profissionais de saúde foi de 50 anos, o impossibilitou a avaliação do dado de cicatriz vacinal de BCG. Relatamos que até 1968 a vacina era utilizada por via oral e só a partir dessa data foi substituída pela BCG, via intradérmica, por este motivo não foi possível a avaliação da cicatriz vacinal. Há ainda a possibilidade que a cicatriz vacinal identificada nos participantes do estudo seja secundária à vacina da varíola9 .

Foi avaliado neste trabalho que os indivíduos TCT positivo/QTF negativo, correspondiam a 32,5\% com níveis de IFN- $\gamma$ detectáveis, porém abaixo do ponto de corte $(0,35 \mathrm{UI} / \mathrm{mL})$. Os dados encontrados da concordância do Quantiferon ${ }^{\circ}$ e do TCT sugerem resultados falsos negativos do IGRA (QTF'), que talvez possa ser corrigido com a padronização de um ponto de corte em populações com alta prevalência da doença. Uma possível explicação para esse fato é a maior especificidade do IGRA, por outro lado, os aspectos socioeconômicos podem interferir na prevalência da $\mathrm{TB}^{10}$.

$O$ grau de concordância razoável $(k=0,24)$ pode ser explicado pela subjetividade do TCT, onde alguns fatores poderiam levar a um resultado falso negativo, tais como: erro técnico na aplicação do TCT e variabilidade de resposta imune devido a fatores intrínsecos ou extrínsecos ${ }^{9}$. Alguns estudos sugerem que se deve pensar em resultado de TCT falso positivo devido a uma resposta cruzada com a vacina $B C \mathrm{~B}^{11}$, assim como a um aumento da área de enduração, fato este registrado em indivíduos que fizeram uso da vacina BCG $^{12}$. Porém no presente estudo não podemos afirmar que esses indivíduos foram imunizados, pois os mesmos tinham idade superior à data em que a vacina passou a fazer parte do calendário vacinal. Por outro lado, em localidades com boas condições socioeconômicas e baixa prevalência da TB, verifica-se concordância superior a $80 \%$ entre os testes TCT e IGRA, o caracteriza a TB como um problema de saúde pública no Brasil ${ }^{13}$.

Nesse trabalho, os parâmetros hematológicos de todos os indivíduos não indicavam processos infecciosos, inflamatórios ou desnutrição. A anergia devido à doença avançada, e imunossupressão podem reduzir a sensibilidade do IGRA, dado que descarta a interferência desta natureza na nossa amostra ${ }^{14}$.

Em um estudo realizado com profissionais de saúde em quatro cidades brasileiras com alta incidência de TB, cujos resultados corroboram com o nosso estudo, foi observado que quando utilizado o valor de corte para o TCT $\geq 10 \mathrm{~mm}$ a concordância entre IGRA e TCT foi de $69 \%(\mathrm{~K}=0,31)$ e o valor de corte $\geq 5 \mathrm{~mm}$ foi de $57 \%$, com índice de $\mathrm{k}=0,22{ }^{15}$.

Outro trabalho realizado na Austrália com 193 profissionais de saúde, média de idade de 35,6 anos e vacinados com BCG, a prevalência de TBIL foi de $88,8 \%$ no TCT e $14,5 \%$ no teste $Q F T^{\circledR}$. A concordância entre os testes mostrou valor de kappa menor que $0,05^{16}$. Similarmente a um estudo de coorte que analisou os testes do IGRA e TB subsequente em 643 contatos de TB, com um seguimento médio de 3,7 anos, a correlação dos resultados dos testes mostrou índice kappa = 0,35 (MOYO et al.,2015). Naranjo e colaboradores (2010) avaliaram uma população heterogênea composta de imigrantes e população local e encontraram os seguintes resultados entre indivíduos contactantes: TCT ( $\geq 10 \mathrm{~mm}$ ) k=0,53 e contatos não bacilíferos TCT ( $\geq 5 \mathrm{~mm}$ ) $\mathrm{k}=0,28$. Os resultados apresentados diferem dos dados encontrados no nosso trabalho. Talvez a explicação para essa diferença seja o fato da heterogeneidade da amostra. Outra pesquisa realizada na mesma área geográfica apresentou resultados diferentes. Em uma amostra composta de 255 indivíduos contactantes com TB, 100 (39,2\%) eram TCT+ / IGRA+ e outros 94 (36,8\%) indivíduos TCT-/IGRA-. De acordo com este estudo $(k=0,53)$, em um cenário endêmico para a tuberculose, os resultados TCT parecem ser mais adequados na decisão de tratar a infecção por TBIL.

Em outro estudo realizado na cidade de Vitória, Espírito Santo, Brasil, foi avaliada a concordância entre os testes TCT e IGRA e a eficácia do IGRA no diagnóstico de nova infecção por MTB, em contatos domiciliares com TB pulmonar. Os contatos que apresentaram TCT negativo foram submetidos a um segundo teste após 8 a 12 semanas. Concluíram que para contactantes expostos recentemente é recomendado o uso dos dois testes e um valor de corte inferior para IGRA, para permitir um acompanhamento mais próximo para a conversão em potencial, $\mathrm{k}=0,57^{17,18}$.

Portanto o risco de transmissão de tuberculose no ambiente de trabalho pode variar principalmente em função da prevalência de doentes no ambiente de trabalho e da efetividade do programa de diagnóstico e controle da infecção da instituição. 


\section{CONCLUSÕES}

O nível de concordância entre o TCT e o QTF mostrou-se razoável $(k=0,24)$. Entretanto, não podemos ainda inferir que existe um padrão-ouro para diagnóstico de TBIL devido às limitações destes testes. Considerando as limitações do TCT em relação a especificidade do QTF sugere-se que a utilização de ambos para a confirmação da TBIL, tendo em vista o aspecto epidemiológico e ocupacional na interpretação destes testes para o uso ou não de quimioprofilaxia, pois as drogas tuberculostáticas apresentam efeitos adversos secundários.

\section{AGRADECIMENTOS}

Agradeço aos participantes da pesquisa, aos pesquisadores do grupo de tuberculose, ao Hospital Especializado Octávio Mangabeira e a Universidade Federal da Bahia.

\section{FINANCIAMENTO}

Esse estudo foi financiado pelo Laboratório de Imunologia e Biologia Molecular / LABIMUNO (ICS/UFBA) e com apoio do Hospital Octavio Mangabeira HEOM (SESAB)

\section{REFERÊNCIAS}

1. BUSATTO, C. et al. Tuberculose ativa versus tuberculose latente: uma revisão de literatura. J. Infect. Control., St. Louis, v. 4, n. 3, 2016.

2. BRASIL. Ministério da Saúde. Secretaria de Vigilância em Saúde. Departamento de Vigilância das Doenças Transmissíveis. Cartilha para - Agente comunitário de saúde: tuberculose. Brasília: Ministério da Saúde, 2017

3. WORLD HEALTH ORGANIZATION. Guidelines on the management of latent tuberculosis infection: the end TB strategy. Geneve: WHO, 2015.

4. BRASIL. Ministério da Saúde. Secretaria de Vigilância em Saúde. Departamento de Vigilância das Doenças Transmissíveis. Técnicas de aplicação e leitura da prova tuberculínica. Brasília: Ministério da Saúde, 2014

5. MORI, T. et al. Specific detection of tuberculosis infection: an interferon-gamma-based assay using new antigens. Am. J. Respir. Crit. Care Med., New York, v. 170, p. 59-64, 2004.

6. SCHABLON, A. et al. Occupational screening for tuberculosis and the use of a borderline zone for interpretation of the IGRA in German health care workers. PLos ONE, San Francisco, v. 9, n. 12, p. e115322, 2014.
7. WORLD HEALTH ORGANIZATION. Global Tuberculosis Report 2018. 2018. ISBN: 978-92-4-156564-6, 2018.

8. DIEL, R. et al. Comparative performance of tuberculin skin test, QuantiFERON-TB-Gold In Tube Assay and T Spot. Test in TB contact investigations for tuberculosis. Chest, Park Ridge, v.135, p.1010-1018, 2009.

9. WORLD HEALTH ORGANIZATION (WHO). Vaccine-preventable diseases: monitoring system: 2006: global summary. WHO, 2006.

10. CASTAÑEDA-HERNÁNDEZ, D. M.; TOBÓN-GARCÍA, D.; RODRÍGUEZMORALES, A. J. Association between tuberculosis incidence and the Human Development Index in 165 countries ofthe world. Rev. peru med. exp. salud publica, Lima, v. 30, n. 4, p. 560-568, 2013.

11. BABAYIGIT, C. et al. Performance of QuantiFERON-TB Gold In-Tube test and Tuberculin Skin Test for diagnosis of latent tuberculosis infection in BCG vaccinated health care workers. Med. sci. monit., Warsaw, v. 20, p. 521-529, 2014.

12. DOOSTI-IRANI, A.; AYUBI, E.; MOSTAFAVI, E. Tuberculin and QuantiFERON-TB-Gold tests for latent tuberculosis: a meta-analysis. Occup. Med., Chicago, v. 66, n. 6, p. 437-445, 2016.

13. AREND, S. M. et al. Detection of active tuberculosis infection by $T$ cell responses to early-secreted antigenic target $6-k D a$ protein and culture filtrate protein 10 . J. Infect. Dis., United States, v. 181, p.1850-1854, May. 2000.

14. PAI, M. et al. Interferon-gamma assays in the Immunodiagnosis of tuberculosis: a systematic review. Lancet, London, v.4, n.12, p.761-776, 2004.

15. SOUZA, F. M. de et al. Comparison of Interferon-y Release Assay to Two Cut-Off Points of Tuberculin Skin Test to Detect Latent Mycobacterium tuberculosis Infection in Primary Health Care Workers. PLos ONE, San Francisco, v.9, n. 8, p.1-21, 2014

16. HUNG, W. T. et al. Prevalence of latent tuberculosis infection in BCG-vaccinated healthcare workers by using an interferon-gamma release assay and the tuberculin skin test in an intermediate tuberculosis burden country. J. Microbiol. Immunol. Infect., Hong Kong, v.48, n.2, p.147-152, 2013.

17. MACHADO, P. C. et al. Comparação do teste cutâneo tuberculínico e do ensaio de liberação de interferon-gama para diagnóstico de tuberculose latente em agentes comunitários de saúde do Sul do Brasil, Rio Grande do Sul, 2012. Epidemiol. Serv. Saúde, Brasília, v. 23, n. 4, p. 675-681, 2014

18. RIBIERO-RODRIGUES, R. et al. Discordance of tuberculin skin test and interferon gamma release assay in recently exposed household contacts of pulmonary TB cases in Brazil. PLos ONE, San Francisco, v.9, n.5, p.1-10, 2014.

Submetido em: 30/09/2019

Aceito em: 07/04/2020 